Int Arch Allergy Immunol 1997;113:387-389

\title{
Author Index, Vol. 113, No. 1-3, 1997
}

International Archives of

Allergya,,d Immunology

Aalberse, R.C. 262 Aberer,W. 105 Achatz, G. 114, 142 Adachi,M. 307 Adamek, L. 355

Adelman, D.C. 45 Adt,M. 200 Akasawa, A. 293, 370 Akitaya,T. 321 Allen, C. 167 Almo,S.C. 109 Anderson, L.R. 373 Annunziato, F. 153 Arruda,L.K. 102 Askenase, P.W. 373 Aubry,J.-P. 329 Austen, K.F. 51,355

Bacher,S. 146 Ball,T. 109 Ballow, M. 167 Barata,L. 196 Baron, J. 173 Baumruker, T. 39 Beauregard, K. 352 Becker, A.B. 58 Becker, W.-M. 69, 118 Befus,A.D. 337 Behrendt, H. 69, 352 Beil,D. 352 Bello,M.G. 297 Benjamin, D.C. 99 Bereziat, G. 339 Berrens, L. 236 Bertrand, C. 231 Bewley,A.P. 131 Bews, J.P.A. 231 Bieber,T. 30 Bischoff, S.C. 348 Bitoh, S. 58, 323 Bjerke, T. 266, 302 Bjòrkstén,B. 80 Blanca,M. 342,345 Blaser,K. 213 Bochner, B.S. 45 Bolsmann, K. 335 Bonnefoy, J.-Y. 329 Braam, U. 335 Breitenbach, M. 114, 125 Breiteneder, H. 105,125, 170 Briza, P. 114, 125 Brodsky,L. 167

Brown, K. 266 Burrows, L.J. 131 Busslinger, M. 35 Byun,D.-G. 157

Capron, A. 48 Capron, M. 48 Capulong, M.C.T. 370 Carson, D.A. 227 Carter, M.D. 96 Carvajal, M.J. 345 Chapman, M.D. 99, 102 Church, M.K. 131 Clough,G.F. 131 Cockcroft, D.W. 269 Coleman, J.W. 316 Corr,M. 227 Corvaïa, N. 266 Cowburn,A.S. 355 Coyle,A. 231 Crameri, R. 213 Crescenzo, G. de 190,200 Creticos, P.S. 224

Daëron, M. 138 Dahinden, C.A. 134 Dahlback, M. 181 Dahlgren,U. 219 Dahlman-Höglund, A. 219 Darsow,U. 379 Daser,A. 150 Daul, C.B. 240 Davison, J.S. 337 DeCarli,M. 153

Delaporte,E. 48 Delespesse, G. 157 D’Hios,M.M. 153 Delneste, Y. 329 Del Prete, G.-F. 153 De Marino, V. 190 Demeure, C.E. 157 De Monte, L. 35 Denburg,J.A. 181 Di Bello,M.G. 297 Dreborg, S. 238 Durham, S.R. 128,196,376

Ebisawa,M. 293

Ebner,C. 105,114, 125,170,246

Eggleston, P.A. 224

Eichler, H.-G. 246

Einsle, K. 231

Elfman,L. 249 Ellis, R. 181 Emadi Khiav, B. 291 Eum,S.-Y. 231

Faith, A. 213 Fedorov, A. A. 109 Ferdinand, S.I. 131 Fernandez, M. 342 Ferrándiz, R. 238

Ferreira,F. 105, 114, 125 Ferrer, M. 305 Fridkin,M. 295 Friedl-Hajek, R. 170 Friedrichs, K.H. 69 Fritzsche, C. 69 Fujita,K. 370

Gai,P. 297 Gajhede,M. 243 Galli,S.J. 14 Gatsios,P. 300 Gauchat, J.-F. 329 Genovese, A. 200 Gfesser,M. 379 Gladis-Villanueva, M.d.M. 335 Gleich,G.J. 200 Glovsky,M.M. 368 Gonzalez, F.J. 342,345 Gordon, J.R. 269 Goto,Y. 323 Gould, H.J. 35,128 Gounni,A.S. 48 Grabbe,J. 284 Graeve, L. 300 Grant, J.A. 376 Greenberg, S.J. 167 Greyerz, S. von 177 Grote,M. 209 Guydon, L. 87

Habre,W. 75 Hales, B.J. 96 Hamann,W. 300 Hamid,Q.A. 128 Hasegawa, S. 323 Hashimoto, K. 323 Hayday, A.C. 373 HayGlass, K.T. 281 Hedin,H. 358 Hemmann, S. 213 Hentschel, M. 384 
kaíigeh

E-Mail karger@karger.ch Fax+ 41613061234 http://www.kargcr.ch

(C)1997S. KargerAG, Basel

387

Henz,B.M. 284

Hershkoviz, R. 295

Hertl,M. 173

Herz,U. 150

Heusser, C.H. 231

Hirani, S. 326

Hirschwehr, R. 114

Hirthenlehner, K. 125,246

Hoffmann-Sommergruber, K. 105

Holgate, S.T. 355, 366

Holmes, BJ. 160,279

Holt,BJ. 75

Holt,P.G. 75

Hong,C.-S. 360

Horowitz, E. 87

Hoshino,A. 272,331

Howarth,P.H. 366

Hsieh,L.S. 105

Humbert, M. 376

I-ijima, H. 323 Iikura, Y. 293, 370 Ikagawa, S. 255 Inman,M.D. 181 Ipsen, H. 243 Ishii,A. 203 Ishikawa, T. 255 Ito, K. 203

Jansen, H.M. 287, 363 Jardieu, P.M. 45, 302 Jeannin, P. 329 Jenmalm, M.C. 252 Jeoung,B.-J. 240 Jones, N. 326 Joyner, D. 87 Juarez, C. 342, 345 Julius, P. 318

Kacy,M. 312 Kagey-Sobotka, A. 224 Kakinuma, Y. 321 Kamal,A. 275 Kaminuma, O. 272,331

Kapp,A. 384 Karlsson, J.-A. 275 Karlsson, M. 219 Kawahara, H. 370 Kawasaki, S. 203

Kay,A,B. 196,376 Kemeny, D.M. 160,279 Kim,C.W. 360 Kimata,M. 293 Kinder, H. 352

Kinet,J.-P. 42 Kips,J. 231 Kochan,J. 302 Kohyama, T. 203 Kokubu,F. 307 Kon,O.M. 376

König,W. 339

Koren,H.S. 65

Koya,N. 370

Kraft, D. 105,114,125,246,258,260

Kricek,F. 216

Krilis, S.A. 289

Kroegel,C. 318

Kunkel,G. 312

Kurishima, K. 323

Laffer, S. 260 Lam, B.K. 355 Lamers, M.C. 142, 146 Lane,S.J. 193 Lang,G.M. 58,323

Larsen,J.N. 243 Lecoanet-Henchoz, S. 329 Lee,D.J. 227 Lee,T.H. 193 Lefort,J. 231 Lehrer,S.B. 122,240 Leiva, L. 345 Lengrand, F. 48 Li,L. 289 Li,X.-H. 269 Li,Y. 281 
Lichtenstein, L.M. 45, 224 Lider,O. 295 Lidholm,J. 249 Life, P. 329 Ljungström, K.-G. 358 Loh,R. 75 Løwenstein, H. 243 Luis, A.M. 236 Lundin,S. 219 Lutter, R. 287, 363 Luttmann,W. 318

MacAry,P.A. 160,279 Macaubas, C. 75 MacDonald, S.M. 187 MacGlashan, D.W., Jr. 45 Maggi, E. 153 Malveaux, F. 87 Manetti, R. 153 Mannaioni, P.F. 297 Manns, M.P. 348 Marino, I. 200 Marone,G. 190,200 Masini,E. 297 Masliah,J. 339 Masuyama, K. 255 Mathison, R.D. 337 Matsukura, S. 307 Matsumoto,T. 370 Matsushita, S. 255 Maurer, D. 24 Mauri-Hellweg, D. 177 Mavilia,C. 153 Mayer, C. 213

Mayer, J. 348 Mayer, P. 55 Mayorga,C. 342 Meier, P.N. 348 Mekori,Y.A. 295 Melchers, F. 11 Meng,Q. 196 Merk,H.F. 173 Metcalfe, D.D. 184 Miescher, S. 216 Mikami,T. 272,331 Mirabella,C. 297 Miyazawa, K. 272 Mizushima, Y. 321 Monticelli, S. 35 Mori, A. 272,331 Mori,K. 293 Morita, M. 48 Mudde,G.C. 266 Mueller, G.A. 99

Nagata,H. 184 Nakagawa, T. 321 Nakajima,T. 293 Natter, S. 209 Neerven, R.J.J. van 243

Nicodemus, C.F. 224, 326 Niederau, D. 173 Nielsen, L.P. 302 Nishimura,Y. 255 Nizankowska, E. 355 Nomura, A. 323 Norman, P.S. 224,326

Obata,T. 370 Oberkofler, H. 114 O’Byrne,P. 181 Ochsensberger, B. 134 Oehling,A. 305

Ohmura,T. 272,331 Ohshima,Y. 157 Ohtoshi,T. 203 Okada,T. 184 Okudaira,H. 272,331

Osmark,P. 243 Otsuka,T. 370 Out, T.A. 287, 363 Owen,M.J. 373 Oya,Y. 370

Pangburn, M.K. 368 Park,J.W. 360 Parmar, J. 366 Parronchi, P. 153 Pastore,A. 246,260 Patella, V. 200 Pater-Huijsen, F.L. de 287 Pauli,G. 249 Pearce,F.L. 291 Peiritsch, S. 266

\section{8}

Int Arch Allergy Immunol Vol. 113, No. 1-3, 1997

Author Index

Pendl,G.G. 39 Penrose,J.F. 51,355 Perdok,G.J. 262 Petersen, L.J. 131 Philip, G. 326 Piccinni, M.-P. 153 Pichler, W.J. 177 Pickart, L. 55 Platts-Mills, T.A.E. 83 Pollart Squillace, S. 83 Pompen, M. 287, 363 Poulsen, F.M. 243 Poulsen,L.K. 302 Preciado-Patt, L. 295 Prescott, S.L. 75 Prieschl, E.E. 39 Prieto,I. 305 Probst, G. 114 Proud, D. 224 Prussin, C. 163 Przybilla,B. 264 Ptak,W. 373

Rajakulasingam, K. 376 Raz,E. 227 Reese, G. 122,240 Reijneke, R.M.R. 287 Reischl, I.G. 266 Rempel,J.D. 281 Renz,H. 150,384 Rich,G. 167 Riemer, M.J. de 287 Rihoux,J.-P. 339 Rihs, S. 134 Ring,J. 7,69,352,379 Romagnani, P. 153 Romagnani, S. 153 Roman, M. 227 Romano, A. 342 Rosenwasser, L.J. 61 Rübben,A. 173 Rudolf, M.P. 216 Rule,G.S. 99

Saito, H. 293, 370 Sakaguchi,N. 293,370 Sakamoto, T. 323 Sakamoto, Y. 370 Sampognaro, S. 153 Sampson, A.P. 355 Santamaria, L.F. 345 Sanz,M.L. 305 Sato,M. 203 Scharenberg, A.M. 42 Scheiner,O. 105, 125, 170

Schierhorn, K. 312 Schiessl,B. 55 Schmutzler, W. 300,335 Schnyder, B. 177 Schou,C. 243 Schunack,W. 297 Schuurman, J. 262 Seder, RA. 163 Sehmi,R. 181 Sehon,A.H. 58 Seiberler, S. 209 Semere, T. 184 Shimada, J. 321 Shoji, S. 203 Shu,U. 157 Shute,J.K. 366 Siemann, U. 170 Sihra,B.S. 376 Simon, B. 114 Simon, H.-U. 206 Simons, F.E.R. 281 Sladek, K. 355 SliwaTomczok, W. 69 Sly,P.D. 75 Smith, A.M. 99,102 Smith, W. 96 Sowka, S. 105,170 Spangfort, M.D. 243 Sperr,W.R. 246 Spiegelberg, H.L. 227 Stadler,B.M. 216 Steinberger, P. 258,260 Steinhart, H. 352 Stingl,G. 24 Strube, D. 264 Stumper, B. 55 Suko,M. 272,331 Svensson, M. 249 Szczeklik,A. 355 Szczepanik, M. 373 
Taborda-Barata, L. 376 Tachimoto, H. 293, 370 Tahara,K. 370 Takafuji,S. 203 Taketomi, EA. 99 Takizawa, H. 203 Telemo,E. 219 Thienes,C.P. 35 Thomas, P. 264 Thomas, W.R. 96 Tighe, H. 227 Togias, A. 45, 87 Tohma,S. 203 Tokunaga, H. 307

Tomczok, J. 69 Torres, MJ. 342 Triggiani, M. 190

Uchida,Y. 323 Unger,A. 114 Ushio,H. 373

Vailes,L.D. 102

Valent,P. 246

Valenta, R. 109, 209, 246, 249, 258, 260

Vanek-Krebitz, M. 105

Vangelista, L. 246,260

Vargaftig, B.B. 231

Venaille, TJ. 75

Vercelli,D. 35

Vieluf,D. 352,379

Vieluf,I. 352

Vogel,M. 216

Vrtala, S. 246

Wagner, K. 231 Wang,M. 281 Ward, PA. 368 Warner, J A. 318 Warner, R.L. 368 Webber, S. 275 Weck,A.L.de 23,55 Welker,P. 284 Wendland,T. 177 Werfel,T. 384 Williams, C.M.M. 316 Withnall,M. 275 Woerly,G. 48 Woisetschläger, M. 266 Wood,L. 181 Wood,RA. 224

Worobec,A,S. 184

Xiang,S. 167

Yabuhara, A. 75 Yang,L.-P. 157 Ying,S. 196 Yun,S.K. 370

Zanni,M. 177 Zeck-Kapp,G. 348 Zhang, M. 312 Zheng, R. 275 Zionchek,K. 302 Zuberbier,T. 284 Zürcher,A.W. 216 Zwadlo-Klarwasser, G. 300, 335

Author Index

Int Arch Allergy Immunol Vol. 113, No. 1-3,1997

389 